\title{
Building a Proposed Conceptual Framework For DDDM in Egyptian Transportation Companies
}

\section{Radwa Mohamed Elsofy \\ Ph.D. Candidate}

(SAMS)
Prof. Mohamed M. El Hadi

Prof. of Computer and Information Systems (SAMS)

\section{Abstract}

Over the last five years, big data and predictive analytics technologies have created new opportunities for companies to advance their decision-making that the ability of making fact based decisions depending on the ability to discover more meaningful data. Data-driven decision making exists at the intersection of data quality and decision quality, where quality data supports quality business decisions. Data-driven decision making is about: Collecting appropriate data, Analyzing that data in a meaningful fashion, Getting the data into the hands of the people who need it, Using the data to increase efficiencies and Communicating data-driven decisions to key stakeholders. Predictive analytics connects data to effective action by drawing reliable conclusions about current conditions and future events. Predictive analytics delivers value to an organization when used to improve decision making. There are three public companies specialized in the public transportation of passengers between regions in Egypt:

1. East Delta for Transportation and Tourism Company.

2. Upper Egypt Transportation and Tourism Company.

3. West and Middle Delta Transport and Tourism Company.

These companies are affiliated to The Holding Company for Maritime and Land Transport which is one of the public enterprise sector companies. These three companies are pioneer in the field of local and international services of transportation and tourism with advantageous levels of services. They aim at maximizing their revenues and improving their competitive advantages. In this paper we will give an overview to the process of data driven decision making and propose a conceptual framework aims to provide a comprehensive picture of the DDDM process in the field of public transportation of passengers between regions in Egypt.

\section{Keywords:}

data-driven decision making (DDDM), big data, Predictive analytics, decision-driven data management.

\section{1-Introduction}

One important and challenging task for managers is to make decisions under our data-rich environment in which decision makers have access to a wealth of information. The simplest definition of data-driven decision making is the use of data analysis to inform courses of action involving policy and procedures (Picciano, 2012). Inherent in this definition is the development of reliable and timely information resources to collect, sort, and analyze the data used in the decision making process.

Decision making in the land transportation companies is an integral component of complex management processes such as operational planning, policy making, and budgeting. These processes evolve over time, require participation by stakeholders, and most importantly, seek to include information which will help all those involved in the decision process.

\section{2-Research framework}

2. 1 Objectives of the research paper:

This research paper aims to:

- Give an overview to the process of data driven decision making.

- Propose a basic conceptual framework aims to provide a comprehensive picture of the DDDM process in the field of public transportation of passengers between regions.

\subsection{Problem statement:}

Although the mentioned three public companies (East Delta, Upper Egypt and West \&Middle Delta) are pioneer in the field of local and international services of transport between regions and have large investments in fleets, workshops for 
maintenance branches and warehousing, they get low profits. Hence this research paper tries to help the managers and planners of those companies to manage their data and get the greatest value from their data warehousing and information delivery systems for making fact based decisions.

\subsection{Previous studies:}

Many researchers have made extensive efforts in implementing data-driven decision making systems in many fields, here are a review of two of dissertations and three of scientific papers from those researches:

The first dissertation was titled "Data-driven modeling and transportation data analytics" This dissertation was submitted by Dali Wei to the Graduate Faculty of Texas Tech University in Partial Fulfillment of the Requirements for the degree of doctor of Philosophy in 2014 (Wei, 2014). This dissertation focuses on investigations of advanced data-driven methods and developing mathematical models and solution algorithms for analyzing multimodal transportation data from a single source, multiple sources, and large networks.

The second dissertation was titled "Data-Driven Analysis \& Policy Implications in the Transportation World: A Focus on Pennsylvania Inspection \& Registration Data and Nationwide Crash Data". This dissertation was submitted by Dana Elyse Peck in partial fulfillment of the requirements for the degree of doctorate of philosophy from Carnegie Mellon University Pittsburgh, Pennsylvania in September 2015 (Peck, 2015). This thesis focuses on various analyses that can be performed pertaining to light duty vehicles by primarily using one, low-cost data source from the annual vehicle safety inspection records in Pennsylvania. It also exemplifies how adding additional, low-cost, data sources can add even more depth and breadth to data-driven transportation analyses.

The first scientific paper under titled "Data and Analytics Data-Driven Business Models: A Blueprint for Innovation: The Competitive Advantage of the New Big Data World" (Brownlow et al., 2015). In this paper the authors presented an integrated framework that could help stimulate an organization to become data-driven by enabling it to construct its own Data-Driven Business Model (DDBM) in coordination with the six fundamental questions for a data-driven business. The six fundamental questions are:

1. What do we want to achieve by using big data?

2. What is our desired offering?

3. What data do we require and how are we going to acquire it?

4. In what ways are we going to process and apply this data?
5. How are we going to monetize it?

6. What are the barriers to us accomplishing our goal? (Brownlow et al., 2015).

The second scientific paper under titled "Decision-Driven Data management: A Strategy for Better Decisions with Better Data". It was a white paper published by SAS in 2012 (Harris, 2012). The purpose of this paper was: examining the following points:

- Variables that can disrupt data-driven decision making, such as time, big data, pattern recognition, human biases, intuitions and errors.

- Why decision-driven data management is a strategy for enabling your organization to achieve better decisions with better data.

- Why closing the decision-data feedback loop is the most critical success factor in data-driven decision making.

The results of this paper refers that implementing data quality allows the organization to:

- Set business rules, monitor progress and maintain data governance policies across applications to gain more control over your data.

- Monitor data-driven decision making in real time or nearreal time, building feedback loops that allow managers to quickly identify issues and make necessary changes when and where they need them.

- Analyze existing applications, data, documents and other artifacts in order to stay on top of the current status of your business and make well-informed, game-changing decisions.

The third scientific paper under titled "Data-Driven Intelligent Transportation Systems: A Survey" it was published in IEEE at 2011 (Zhang et al., 2011). In this work a survey has been provided on the development of data-driven intelligent transportation systems, discussing the functionality of its key components and some deployment issues associated with data-driven intelligent transportation systems. Future research directions for the development of data-driven intelligent transportation systems were also presented.

\subsection{Methodology:}

This work employs a case study method concerning the three public enterprise sector companies specialized in the public transportation of passengers between regions in Egypt. Therefore, a descriptive and analytic research method is being followed. This is depending on the following data gathering techniques: 
- Questionnaires technique as the most important one to get the data from users, it is described as the cheapest tool for usability.

- Interview technique that uses formal face-to-face meetings to determine which facts or data that were collected are relevant (Modell, 2007).

- Literature review regarding the different facts of the work.

\section{Data-Driven Decision-Making Overview:}

The simplest definition of data-driven decision making is the use of data analysis to inform courses of action involving policy and procedures (Picciano, 2012).

Data-driven decision making is about (Sagebrush, 2004):

- Collecting appropriate data.

- Analyzing that data in a meaningful fashion.

- Getting the data into the hands of the people who need it.

- Using the data to increase efficiencies.

- Communicating data-driven decisions to key stakeholders.

To answer the question of: How Does Data-Driven Decision making affect Firm Performance? An academic research conducted at 2011 examined whether firms that emphasize decision making based on data and business analytics (DDD) show higher performance. Using detailed survey data on the business practices and information technology investments of 179 large publicly traded firms, they found that firms that adopt DDD have output and productivity that is $56 \%$ higher than what would be expected given their other investments and information technology usage. Furthermore, the relationship between DDD and performance also appears in other performance measures such as asset utilization, return on equity and market value (Brynjolfsson, E., Hitt, L., and Kim, H., 2011).

\subsection{Decision Lifecycle:}

There are many challenges to delivering a comprehensive and integrated decision making environment. These challenges prevent the flow of information across the different phases within the decision. SAS whitepaper suggests the decision life cycle illustrated below in (figure 1) for making an optimal decision. This lifecycle consists of four steps (discover, model, deploy and monitor) (SAS, 2014).

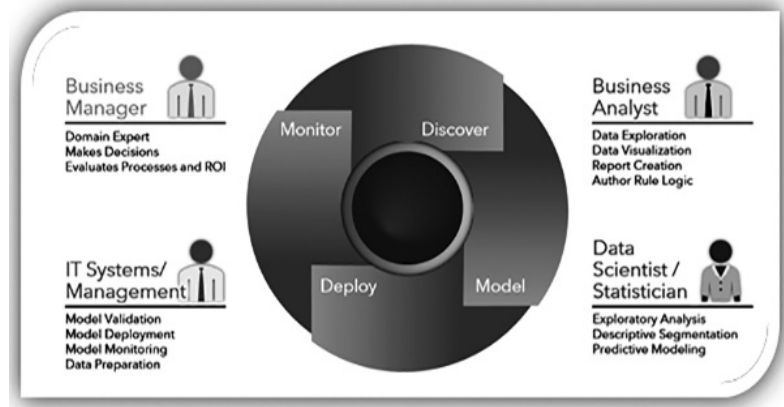

Figure 1: Decision Lifecycle (SAS, 2014)

\subsection{Decision-Driven Data Management:}

For producing a successful decision the managers need to manage their data and put the three components of decision driven data management in the top priority position. The three components of decision-driven data management are: (Harris, 2012):

\section{Decision Criteria:}

Decision-driven data management begins with the decision in mind in order to decide the organization's decision support needs.

Decision criteria, includes (Harris, 2012):

- Time constraints and preferences: Time constraints often trigger time-inconsistent data quality preferences, which provide a framing effect for decision criteria that cannot be overstated. For example, a decision that must be made within 30 seconds has very different data requirements than a decision that should be made within 30 minutes, or a decision that could be made within 30 days.

-Business impacts and risks: The business impacts associated with a decision will vary, as will the potential risks associated with making a bad decision.

- Human intuitions and biases: Documenting any potential pre-decision or post-decision data bias will help better evaluate the effectiveness of companies' data requirements, as well as help better evaluate the business results produced by that decision.

\section{Data Requirements:}

Different decisions will have different data requirements, which include the data volume, variety and velocity necessary for data-driven decision making. This aligns data management and governance activities for better decision support, and establishes decision-specific data quality thresholds.

Better decisions come from better data quality thresholds, which deliver better data to the decisions that require it and 
provide a business justification for data quality improvements that will improve business performance.

\section{Decision Evaluation:}

The quality of a decision is determined by the business results it produces. Even though evaluating decision quality may only establish a correlation, and not causation, between the decision execution and its business results, it is still essential to regularly and systematically evaluate your decisions.

Every decision, even an intuition, produces data that can be used as the basis for improvement (Harris, 2012).

(Figure 2) below illustrates the Decision-driven data management components with the feedback loop.
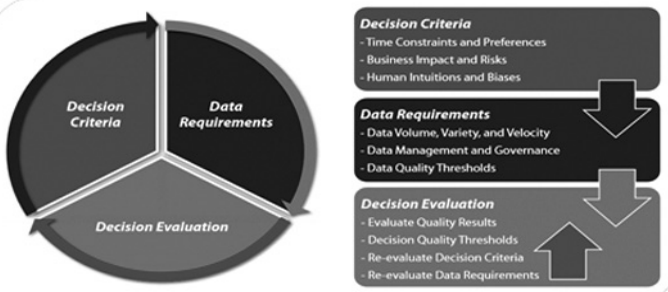

Figure 2: Decision-driven data management components with feedback Loop (Harris, 2012)

\section{Data-Driven Decision Making Process:}

There are many views for utilizing the process of DDDM to help the decision makers for managing their data here are explanation of some of them:

1. (Picciano, 2012) describe Simon DDDM as shown in the following figure:

It assumes that an information system is available to support the decision process, that internal and external factors not available through the information system are considered, and that a course or courses of action are determined.

The information system in (Figure 3 ) is a computerized database system capable of storing, manipulating, and providing reports from a wide variety of data. Terms related to datadriven decision making include data warehousing, data mining, and data disaggregation.

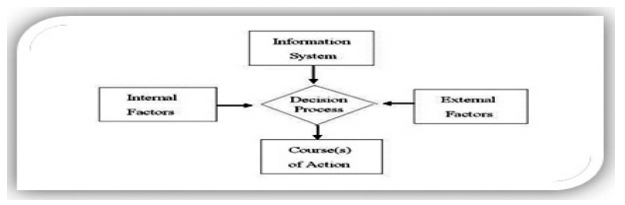

Figure 3: the Data-Driven Decision-Making Process

(Picciano, 2012)

\section{The Data to Decisions framework proposed by (Bruner, 2012) consists of five steps as follows:}

1. Understand what the real business question is: Ask the stakeholder what she really wants to know. "Why is this person asking this question? What's the context, what are the impacted segments?"

2. Create an analysis plan with hypotheses: "What are your fundamental beliefs about this problem? Let's do a guided exploration. This is where you decide what methodology you're going to use--am I going to use correlation analysis, is this a profiling problem, am I going to go into predictive analysis?"

3. Collect data: Based on step 2, you'll know what sort of data you need to collect.

4. Gather insights: "Now that you've collected data, you've ordered it, validated it, triangulated it, then you get into the analysis part based on the technique you've chosen."

5. Make recommendations. "It's not only the technical aspects that are important, but also the soft aspects: what are you doing with your stakeholders? You are an analyst. What are you doing with the folks on the other side? How are you building alignment? If you've not brought your stakeholders along, then they won't necessarily take your recommendations."

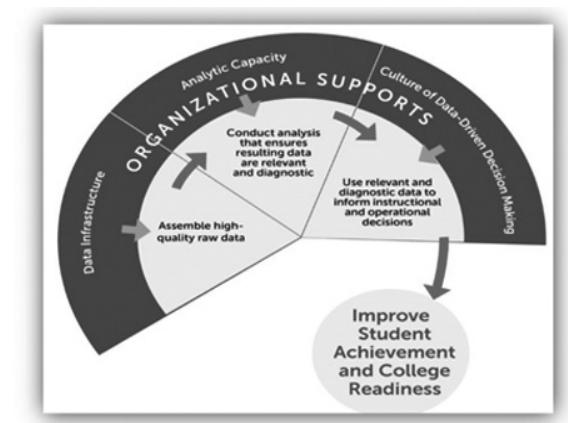

Figure 4: DDDM theory of action and organizational supports (Gill, B.,\& Borden, B.\& Hallgren, K., 2014)

3. (Gill, B., \& Borden, B. \& Hallgren, K., 2014):

Provides a comprehensive picture of the DDDM process in education by providing two frameworks The first of the two framework diagrams (Figure 4) provides a high-level, generalized view of a theory of action-a causal chain-for how DDDM can lead to improved student achievement. The second framework divided into two diagrams the first one illustrated the Decision makers and data uses while the second diagram illustrated the different kinds of data that are relevant for decision makers at different levels of the education system. 


\section{4. (Marr, 2016) broken down DDDM process into a simple ten-steps as follows:}

1. Start with strategy: It's easy to get lost in our big data environment. Hence start by what your business is looking to achieve instead of starting with what data you could or should access. (Marr, 2016)

2. Hone in on the business area: Identify which business areas are most important to achieving overall strategy of the enterprise such as the customer, finance and operations.

3. Identify your unanswered business questions: Now that you've identified your strategic objectives, the next step is to work out which questions you need to answer in order to achieve those goals. By working out exactly what you need to know, you can focus on the data that you really need. Your data requirements, cost and stress levels are massively reduced when you move from 'collect everything just in case' to 'collect and measure $\mathrm{x}$ and $\mathrm{y}$ to answer question $\mathrm{z}$ '.

4. Find the data to answer your questions: The next step is to identify what data you need to access to answer these questions. It's really important to understand that no type of data is inherently better or more valuable than any other type. Focus on identifying the data that could help you answer your most pressing questions and deliver on your strategic objectives. Make a note of which data sets you could use to answer those questions. You can then choose the best data options to pursue based on how easy the data is to collect, how quick and how cost effective it is.

5. Identify what data you already have: Once you've identified the data you need, it makes sense to see if you're already sitting on some of that information, even if it isn't immediately obvious. Internal data accounts for everything your business currently has or could access. If the data doesn't already exist, then find ways of collecting it either by putting data collection systems in place or by acquiring or accessing external data.

6. Identify your unanswered business questions: Once you know the costs, you can work out if the tangible benefits outweigh those costs. In this respect, you should treat data like any other key business investment. You need to make a clear case for the investment that outlines the long-term value of data to the business strategy. If you believe the costs outweigh the benefits, then you may need to look at alternative data sources.

7. Collect the data: Much of this step comes down to setting up the processes and people who will gather and manage your data. You may be buying access to an analysis- ready data set, in which case there's no need to collect data as such. But, in reality, many data projects require some amount of data collection.

8. Analyze the data: You need to analyze the data in order to extract meaningful and useful business insights. The most common types of analytics are text analytics, speech analytics and video/image analytics. The past few years have seen an explosion in the number of platforms available for big data analysis. Some platforms require nothing more than a working knowledge of Excel, meaning most employees can dip their toes into big data analysis.

9. Present and distribute the insights: Unless the results are presented to the right people at the right time in a meaningful way then the size of the data sets or the sophistication of the analytics tools won't really matter. You need to make sure the insights gained from your data are used to inform decision making and, ultimately, improve performance.

10. Corporate the learning into the business: Finally, you need to apply the insights from the data to your decision making, making the decisions that will transform your business for the better.

From the previous views we can conclude that to be a DDDM organization you need to:

- Start from your strategic goal, identify the needed data to achieve this goal, try to collect and analyze those data instead of getting lost in our massive amounts of available data and take actions.

- It is important to use the suitable data analytical tool.

- Relevant and diagnostic data has to be presented to the right decision makers at the right time in a meaningful way to improve decision making process.

\section{Case Study:}

East Delta, Upper Egypt and West \&Middle Delta are the three companies specialized in the public transportation of passengers between regions in Egypt. These companies are affiliated to The Holding Company for Maritime and Land Transport which is one of the public enterprise sector companies. These three companies are pioneer in the field of local and international services of transportation and tourism with advantageous levels of services and proficient operation along with perpetual development with the aim of customers comfort and safety. They aim at maximizing their revenues and improving their competitive advantages.

Here are some basic information about these companies: 


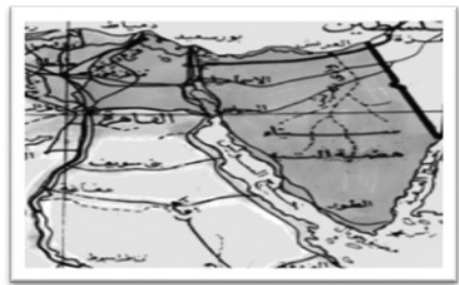

Figure 5: East Delta Company coverage are (Holding Company, 2006)

1. East Delta for transportation and Tourism Company:

- Incorporation date: 1965.

- Steady increase of workers until reaching 6800

- Number of branches: 14.

- Number of operation centers: 20 .

- Number of central workshops: 4 apart from branches' workshops for maintenance.

- Fleet: 800 vehicles.

Figure 5 shows East Delta Company coverage area.

2. Upper Egypt transportation and Tourism Company:

It was founded according to the resolution of the ministry of transport number 14 for 1979. The company head office is located at Nasr city- Cairo in addition to other seventeen branches distributed across three sectors figure 6 shows Upper Egypt Company coverage area.

Authorized capital 60,000 million pounds

Capital and paid 4736258047 million pounds

Fleet 533vehicles

Lines 232 line

employees 4700 approximately

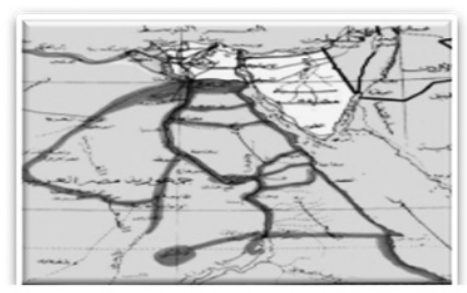

Figure 6: Upper Egypt Company coverage area (Holding Company, 2006)

3. West and Middle Delta transport and Tourism Company:

The company started its activity since the 1950s of the last century as the private sector and then in 1961 as one of the bodies belonging to the Ministry of Transport and then as a sector in the year 1979 and then as a public enterprise sector of commercial feature in accordance with the law 203 of 91 . The company has joined Delta in the Central West Delta bus company with effect from April 16, 2005 to become the new name of the company (bus company West and Central Delta Transport and Touris $\mathrm{m}$ ) and is since that date, said a large doubled with his corps of fleet-service includes many diverse lines permeates the provinces of the Central Delta and West Delta on breadth, population density and thus contribute new company's new format with its expertise and the technical and administrative through unite their efforts and reorganization of the rise in the level of services provided to different categories of passengers and tastes in the passenger transport market(Holding Company, 2006).

- The capital of the company currently: 83.174 million pounds.

- The number of company branches (18).

- The number of lines: 209 line.

Figure 7 shows West and Middle Delta Company coverage area.

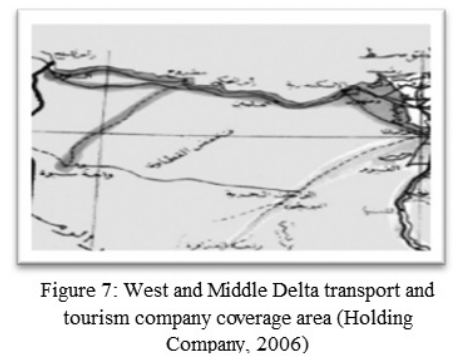

Data Gathering techniques:

- An interview was conducted with three of the board members of the Upper Egypt Company (IT general manager, general manager of planning and transportation sector manager).

- A Questionnaire form is developed specifically for this study to define the transport decision makers' needs and their problems with current data.

The distributed questionnaire covered all the branches of the Upper Egypt Company and all the levels of decision making.

Sampling of the Audience for the Distribution of this Questionnaire:

The sample consist of all the managers of the branches and all the operational managers all over the 17 branches of the Upper Egypt Company. Figure 8 illustrates Audiences and their Responses to the Questionnaire. The 
collected data from this practical study are summarized in table $\mathrm{A}$ and table $\mathrm{B}$.

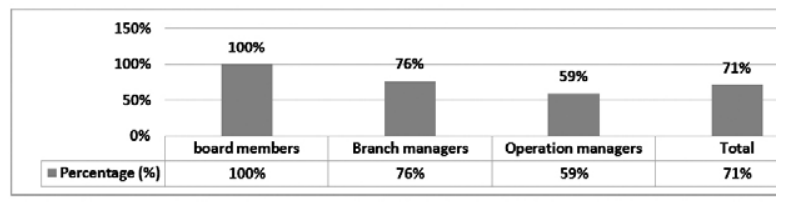

Figure 8: Audiences and their Responses to the Questionnaire

6. Building a Proposed Conceptual Framework for DDDM in Egyptian Transportation Companies

Our proposed DDDM framework depending on the theoretical study for utilizing data in decision making process and the practical study to the decision making process in the public transportation companies.

IN this framework we begin with the decision in mind then try to understand the decision criteria before evaluating the data requirements. The target of the transport companies is to improve the bus revenues.

The proposed framework involves three sequential steps that explains how the data can be used to support the ultimate goal which is supposed to be improving bus revenues and the requirements needed for making an effective data use possible. The three steps are illustrated in (Figure A). The proposed framework supported by two tables (table A: Decision makers and data uses \& Table B: Relevant Needed Data).

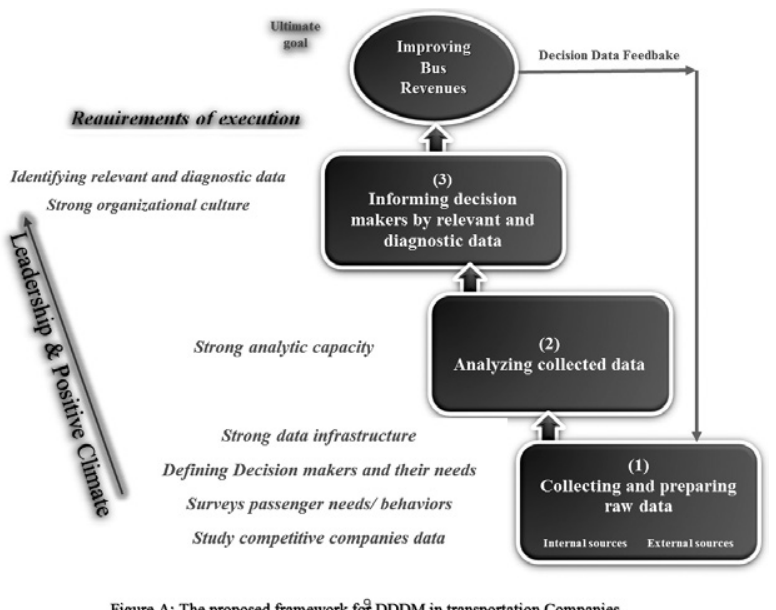

The basic steps for data-driven decision making in transportation companies:

Step1: Collecting and preparing raw data:

We start by the goal (improve bus revenues) and try to identify the needed data to achieve this goal by conducting interviews and made a survey with all the managers and decision makers related to the revenues of the bus. We found that we need detail data about five aspects these aspects are illustrated in figure 9 (bus status, drivers and collectors, operation lines and passengers and competitive companies). The available internal data covers all these aspects except passengers and competitive companies, so we need to collect data about them from external sources.

The basic steps for data-driven decision making in transportation companies:

Step1: Collecting and preparing raw data:

We start by the goal (improve bus revenues) and try to identify the needed data to achieve this goal by conducting interviews and made a survey with all the managers and decision makers related to the revenues of the bus. We found that we need detail data about five aspects these aspects are illustrated in figure 9 (bus status, drivers and collectors, operation lines and passengers and competitive companies). The available internal data covers all these aspects except passengers and competitive companies, so we need to collect data about them from external sources.

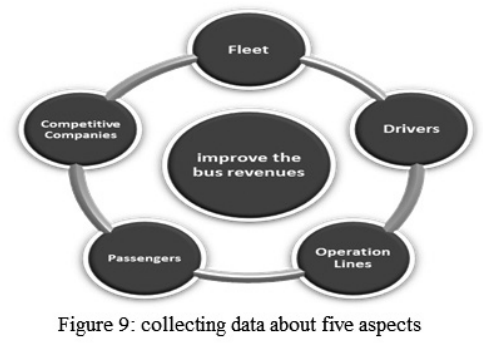

Collected data:

1. Internal sources such as:

- The daily and weekly operational reports.

- Current databases reports: (Human resource - revenue \& operating and depreciation - inventory - purchases Medicare - accidents - automated booking - licenses).

2. External sources such as:

- Surveys investigation of the community members to study and analyze the passenger's behavior and their needs. As these transportation needs are better understood, organizations will have increasing opportunities to provide targeted transportation services.

- Analyzing traveler's opinion posted in social Media.

- The data of the other competitive companies.

- The weather data. 


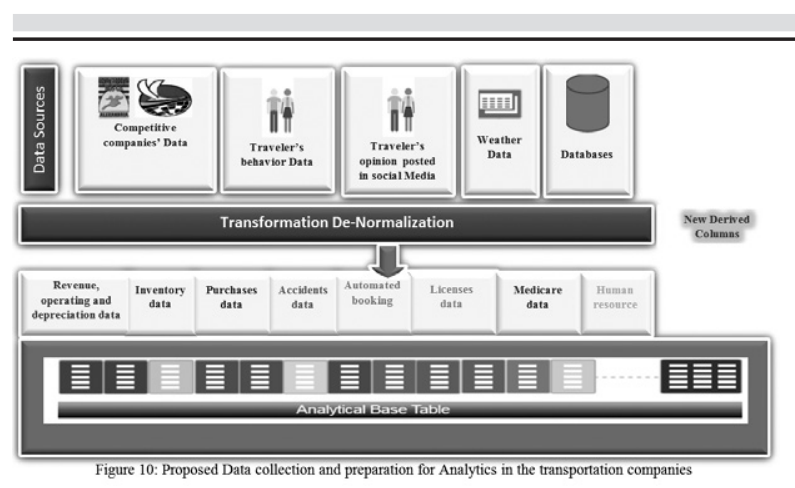

Data Preparation:

Preparing data for analytics requires merging, transforming, and de-normalizing source data from multiple tables into one analytical base table (ABT) (SAS white paper, 2015). Figure 10 shows the proposed Data collection and preparation for Analytics in the transportation companies. Upper Egypt Company.

This step requires:

1. Strong data infrastructure:

The improvement of data systems are essential to the companies' ability to effectively collect, transfer, and manipulate information. Data infrastructure development includes:

- Improvement of technical hardware (servers, computers, peripheral devices, and Internet connections).

- Establishing linkages between distinct databases (linking drivers and Ticket collector data) with (revenues \& operating and depreciation), linking (financial data) with (operational data) facilitates analyses that require connections across data types.

- Creating data collection mechanisms by developing standardized procedures for the collection of data from internal and external sources and storage of this data.

- Adjusting data access and management practices to ensure timely delivery of data to decision makers enhances their ability to make use of the data. Figure 11 illustrates one of the proposed options to improve current IT infrastructure.

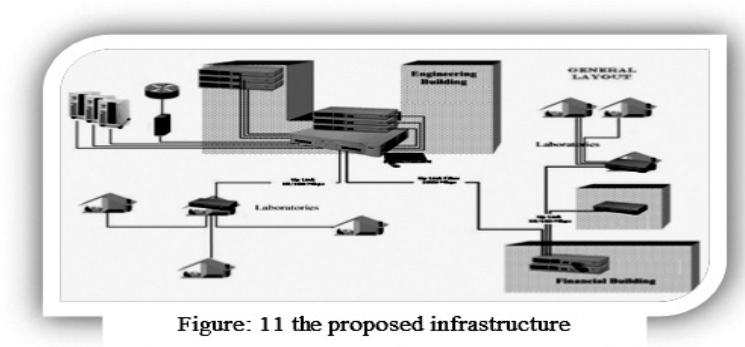

2. Study decision makers and their data needs:

Meaningful use of data begins with who will access, analyze, or review the data and for what purpose it will be used. According to the interview conducted and the analysis of the collected data, this is a list of the different users' levels of data, and the purposes for which they might conduct data analyses which are presented in (Table A).

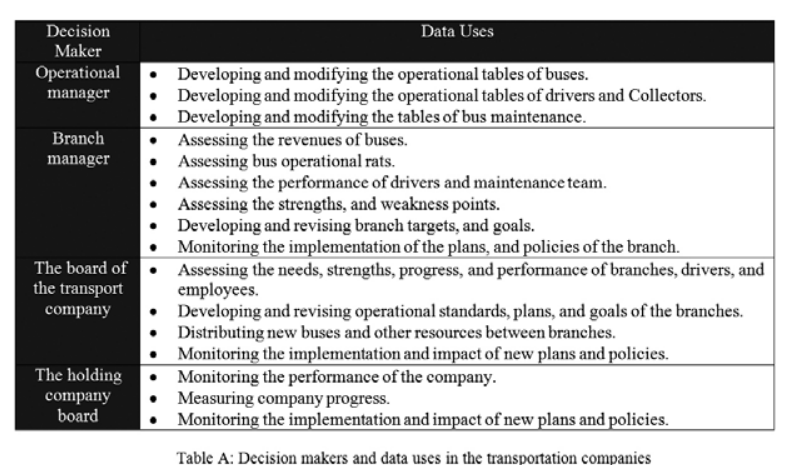

3. Study passengers' needs and their behaviors:

Collecting data about the passengers requires surveying their opinion about the level of the service, their satisfactions, problems, suggestions and behavior and other points. And follow their opinion on the social media.

4. Study competitive companies' data:

Collecting data about the other competitive companies is too important to cope with their offers and services.

Step 2: Analyzing collected data:

Conducting data analysis to ensure that resulting data are relevant and diagnostic is too important. If data are to serve decision making process that ultimately improves bus revenues, they must be relevant to those decision makers and appropriately diagnostic for the decision at hand. Big data (predictive analytics) has significant potential for transportation systems. The last researches conducted by Ventana Research shows that Predictive Analytics is the most popular area of big data analytics as shows in figure 12 (Menninger, 2017). Predictive Models have many usage in transportation field for example:

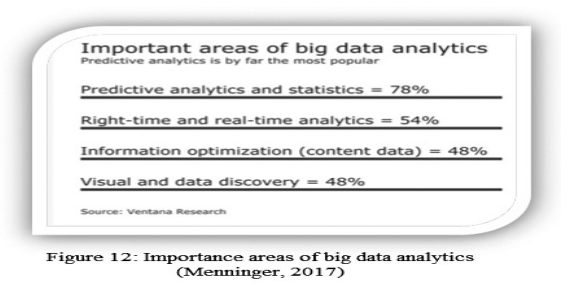


1. In the optimization of public transportation operating costs, and improving public transportation services: Models for predict bus usage was built such as (Gaussian process-based predictive model) this model uses contexts, e.g., time day of the week, hour and rain information to predict bus ridership. It was built in Zurich, Switzerland (Bhattachara, 2013).

2. In predicting bus arrival times: A set of regression models that estimate arrival times for buses traveling between two points along a route was developed by Patnaik et al. These models was built to assist passengers' decision-making by providing passengers with accurate bus arrival information through Advanced Traveler Information Systems. The data applied for developing the proposed model were collected by Automatic Passenger Counters installed on buses operated by a transit agency in the northeast region of the United States (Patnaik et al., 2004).

3. In Response/Purchase: Transportation companies earn profits through providing services. Models that predict the response to a low-rate offer have been used in these companies. Response/purchase model scores are used to increase the number of responders/buyers, reduce expenses, or both.

4. In Asset Maintenance: Transportation systems (rail, air, truck, and auto fleet) use predictive models to estimate the likelihood of system interruptions and mechanical failures based on patterns of past events. This allows them to optimize the location of maintenance service and parts as well as assist in labor planning.

5. In Human Resources: In Transportation companies optimal staffing is a main driver of profitability.

This step requires strong analytic capacity by:

- Implementing an effective predictive models for:

o In operational plan of buses.

o Estimating the likelihood of mechanical failures.

0 In the optimization of operating costs, and improving services.

o Predicting bus arrival times.

o Attracting more customers.

By using SAS Enterprise Miner that it can construct and compare accurate and robust statistical and machine learning models.

- Providing training to staff at all levels to increase their individual capacity to access and use data.

- Improving the accessibility of data to enhance the ability of decision makers at all levels to access and use data in a timely manner.

Step 3: Informing decision makers by relevant and diagnostic data: Even the best data and the best analysis will not improve outcomes if the results are not used. A culture of data use is neces- sary to ensure that (relevant, diagnostic) data are not filed away and forgotten. Building feedback loops that allow managers to quickly identify issues and make necessary changes when and where they need them is too important.

This step requires:

1. Identifying relevant and diagnostic data:

In order to guide the improvement of practice data must be relevant to the practice of the particular decision maker and diagnostic for the issue at hand. Irrelevant data will not be used, and non-diagnostic data might be used inappropriately.

Two factors that might be considered in assessing the relevance of the data and the distinctions between diagnostic and non-diagnostic data:

1. Are the data relevant to the decision maker and the decision at hand?

For each level of decision maker, the relevance of data may depend on how frequently the data are updated and delivered; and the level of detail or aggregation.

Decision makers at higher levels of the system typically need data that are aggregated at larger units of analysis and their decisions often do not require data that are updated as rapidly and frequently.

2. Are the data diagnostic for informing improved decisions? The relevance of the data to the decision maker and the decision at hand is not sufficient to ensure that the data will move the decision maker in a productive direction but if not analyzed carefully, they could lead to bad inferences about the branch's performance and bad decisions about how to improve the branch's performance.

(Table B) presents factors that might be considered in assessing the relevance of the data and the distinctions between diagnostic and non-diagnostic data.

2. Strong organizational culture of DDDM:

Strong leadership and systems of accountability may facilitate successful data use. Promoting data sharing encourages staff to openly discuss and reflect on their data.

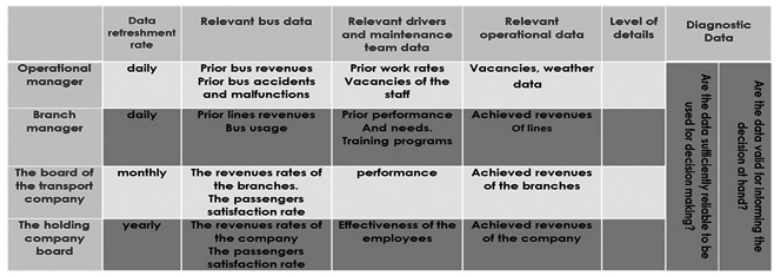

Table B: Relevant Needed Data

At the end leadership and positive climate are too important conditions for successful implementation of data driven decision making process. 


\section{Conclusion:}

- Data-driven decision making is the use of data analysis to inform courses of action involving policy and procedures.

- Decision-driven data management has three components which are: Decision criteria, Data requirements and Decision evaluation. It begins with the decision in mind.

- Data quality thresholds deliver better data to the decisions that require it.

- The quality of a decision is determined by its business results.

- Continuous improvement enables better decisions with better data.

- Data-driven decision making requires decision-driven data management, which uses data quality and data governance as a strategy for enabling your organization to achieve better decisions.

- The creation and improvement of data systems are essential to the companies' ability to effectively collect, transfer, and manipulate information.

- Decision lifecycle consists of four steps (discover, model, deploy and monitor).

- The three sequential steps for the proposed DDDM framework are:

1. Collecting and preparing raw data.

2. Analyzing collected data.

3. Informing decision makers by relevant and diagnostic data.

- Transportation data can be collected from internal sources such as (daily, weekly operational reports, bus database, maintenance database, transactional databases ...) and external sources such as surveys investigation of the community members.

- Meaningful use of data begins with who will access, analyze, or review the data and for what purpose.

- Data must be relevant to the practice of the particular decision maker and diagnostic for the issue at hand.

- Data that are not relevant to the decision makers (for instance, because they arrive too late to inform a decision) will not be used; data that are not diagnostic of the issue at hand may be used in ways that are counterproductive.

- Being driven by data requires much more than the existence of an effective data infrastructure, the accessibility of the data, and a culture of data use. It also requires careful attention to ensuring that data are both relevant and diagnostic for each decision maker and decision. Otherwise, there is a high risk that decision maker will either drive in the wrong direction or drown in the data.

-When transportation system users can be understood and treated more as customers, a world of possibilities for engaging them and influencing their behavior opens up.

\section{References:}

Bhattachara, S., Phithakkitnukoon, S. \& Nurmi, P. (Eds.). (2013). Gaussian ProcessBased Predictive Modeling for Bus Ridership, UbiComp '13 Adjunct, Sept 8-12, 2013, Zurich, Switzerland. ACM 978-1-4503-2139-6/13/09.

Brynjolfsson, E., Hitt, L., \& Kim, H. (Eds.). (2011). Strength in Numbers: How Does Data-Driven Decision making Affect Firm Performance? Retrieved April 3, 2017 from http://dx.doi.org/10.2139/ssrn. 1819486

Bruner, J., (2012). Contributor Five Steps For Making Data-Driven Decisions, Retrieved Jun 3, 2017 from http://www.forbes.com/sites/jonbruner/2012/04/20/fivesteps-for-making-data-driven-decisions/\#7807117b54ed.

Brownlow, J., Zaki, M., Neely, A. \& Urmetzer, F. (Eds.). (2015). Data and Analytics - Data-Driven Business Models: A Blueprint for Innovation: The Competitive Advantage of the New Big Data World, Cambridge Service Alliance.

Gill, B., Borden, B. \& Hallgren, K. (Eds.). (2014). A Conceptual Framework for Data Driven Decision Making, Mathematic Policy Research.

Harris, J. (2012). Decision-Driven Data Management: A Strategy for Better Decisions with Better Data, SAS white paper.

Holding Company. (2006). Upper Egypt Bus Company. Retrieved Jun 3, 2017, from http://bus.com.eg/EBus/OnlineServicesEn/Home/upperegypt/index.aspx

IBM Corporation. (2010). Real world predictive analytics Putting analysis into action for visible results, Author.

Peck, D. (2015). Driven Analysis \& Policy Implications in the Transportation World A Focus on Pennsylvania Inspection \& Registration Data and Nationwide Crash Data (Doctoral dissertation, Carnegie Mellon University Pittsburgh, Pennsylvania). Retrieved Jun 3, 2017from http://repository.cmu.edu/dissertations/640

Picciano, A. (2012). The Evolution of Big Data and Learning Analytics in American Higher Education, Journal of Asynchronous Learning Networks, Volume 16: Issue 3

Marr, B. (2016). Data-Driven Decision Making: 10 Simple Steps: For Any Business. Retrieved Jun 3, 2017 from http://www.forbes.com/sites/bernardmarr/2016/06/14/data-driven-decision-making-10-simple-steps-for-any-business/2/\#7b6682bc6e35.

Menninger, D. (2017). Opinion Big data focus should be on analytics, awareness anticipation and action. Retrieved Jun 1, 2017 from https://www.information-management.com/opinion/data-science-gets-chic

Modell, M., E. (2007). A Professional's guide to systems analysis. Retrieved May 30 2017 from http://www.martymodell.com/pgsa2/pgsa07.html

SAS Whitepaper. (2015). Thirsting for Insight? Quench It With 5 Data Management for Analytics Best Practices. Author.

SAS Whitepaper. (2014). From Data to Decision: How SAS Decision Manager Automates Operational Decisions. Author.

Soy, S. K. (1997). The case study as a research method. Unpublished paper, University of Texas, Austin. Retrieved Jun 3, 2017 from http://www.ischool.utexas. edu/ ssoy/usesusers/l391d1b.htm.

Wei, D. (2014). Data-Driven Modeling and Transportation Data Analytics (PHD's thesis). Texas Tech University, Texas.

Yong, Yin, Kalu, I., Tang, J.\& Zhu, J. (Eds.). (2011). Data mining concepts, methods and applications in management and engineering design, Spring virlag London Limited.

Zwass, V. (2016). Information system. Encyclopedia Britannica. Retrieved May 30 2017 from https://www.britannica.com/topic/information-system.

Zhang, J., Wang F., Wang, K., Lin, W., Xu, X., \& Chen, C. (Eds.). (2011). Data-Driven Intelligent Transportation Systems: A Survey. IEEE Transactions on Intelligent Transportation Systems, Vol. 12, No. 4, December 2011. 